\title{
Gastroenterological Society of Australia
}

The third annual general meeting was held at the Princess Margaret Hospital for Children, Perth, on 4 May 1961.

President

Vice-President

Hon. Secretary

Hon. Treasurer

Other members of council: Sir William Morrow, Dr. S. J. M. Goulston, Dr. W. Irwin.

After the business meeting, a scientific meeting was held.

R. A. JOSKE and D. H. CURNOW outlined the history and metabolic basis of the d-xylose absorption test; the normal value of the test in an Australian population was found to be $6.6 \pm 1.4 \mathrm{~g}$. $/ 5 \mathrm{hr}$. The lower limit of normal was then arbitrarily taken as $3.8 \mathrm{~g} . / 5 \mathrm{hr}$. (= mean 2SD.). Analysis of 200 xylose tests in various disease states showed that a patient with low xylose absorption usually presents other evidence of malabsorption if this is sought, although any degree of malabsorption may occur with normal xylose absorption. Xylose absorption was unrelated to fat, protein, folic acid, $\mathrm{B}_{12}, \mathrm{Ca}$, or $\mathrm{K}$ metabolism. The xylose test was low in atrophic jejunitis and infective enteritis, variable in post-gastrectomy syndromes, the reticuloses and vascular disease, and usually normal in pancreatic disease.

J. B. BLACKWELL described two patients with 'Malabsorption in the presence of primary carcinoma of the small bowel'. Neither had any previous history suggesting an absorptive defect.

The first case was a man, aged 72 years, who had persistently vomited for eight weeks before laparotomy at which a constricting adenocarcinoma of the midjejunum was excised. In the small bowel adjacent to the tumour histological changes of atrophic jejunitis were found. The villi were shortened and fused, the lamina propria inflamed, and pigment granules were prominent in the muscle layer. Clumping and segmentation were demonstrated radiologically, and biochemically a low plasma carotene, low serum albumin, low xylose absorption, and raised faecal fat confirmed malabsorption.

The second case was a man, aged 57 years, who also presented with subacute obstruction caused by an anaplastic carcinoma arising in the lower jejunum. Atrophic jejunitis with complete loss of villi was found in the proximal segment and was present, but to a lesser degree, distally. Malabsorption was considered to be present on radiological evidence and on the biochemical findings of low plasma carotene and potassium values, and low serum albumin and calcium. Faecal fat was $26 \%$.

In a review of 12 other cases of small bowel carcinoma from the hospital files mild changes of atrophic jejunitis were found in two.

Dr. Blackwell suggested that the association of small bowel carcinoma, atrophic jejunitis, and malabsorption may be more frequent than is at present suspected.

D. W. PIPER presented a paper on 'Anticholinergic drugs and gastric secretion', in which he compared a series of new anticholinergic agents with atropine, regarding their effect on saliva flow and gastric secretion and the effect of anticholinergic drugs in general on the various constitutents of gastric juice. All anticholinergic drugs tested, including atropine, have a greater inhibitory effect on gastric secretion than they have on saliva flow. Of the drugs studied (atropine, propantheline, oxyphencyclimine, and proprionyl atropine methyl nitrate), only oxyphencyclimine and propantheline had a selective action on the stomach superior to atropine.

When the effect of anticholinergic drugs was studied on the electrolyte content of gastric juice, a progressive drop in acid output with decreasing volume of secretion was found; the acid concentration showed a less marked fall. With increasing anticholinergic suppression of secretion the sodium and potassium output fell, the potassium output falling to a greater extent than the sodium output. The potassium concentration remained relatively fixed or showed a slight fall with decreasing volume of secretion, while the sodium concentration rose. When the effect of anticholinergic drugs on the mucus content of gastric juice was studied, it was found that increasing anticholinergic suppression was accompanied by a rising mucus concentration; in many cases the total output of mucus showed little change in output with the decrease in secretory volume.

J. G. RANKIN, M. R. PLAyoust, and R. W. BEAL described their studies on the use of $\mathbf{P}^{\mathbf{3}}$-labelled chronic phosphate for the measurement of liver blood flow. The rate, efficiency of hepatic extraction, and sites of removal of subcritical doses of colloidal $\mathbf{P}^{32}$-labelled chronic phosphate from the blood stream were studied in 30 patients with normal and abnormal liver function.

Values for $t \frac{1}{2}$ were similar to those obtained by other workers, but the efficiency of hepatic extraction was found to have a roughly linear relation to the degree of hepatic dysfunction as measured by bromsulphalein retention and to range from $40 \%$ to $93 \%$.

In the presence of normal liver function the liver and spleen together removed approximately all of the administered dose of colloid whereas in cirrhotic patients there was a relative alteration in phagocytic activity of the various organs so that the marrow removed as much as $60 \%$ and the liver and spleen together as little as $26 \%$ to $56 \%$.

The presence of a significantly large extrasplanchnic 
site of removal of subcritical doses of colloid in patients with chronic liver disease invalidates methods using these substances for measuring liver blood flow under these conditions.

A. R. COOKE and A. P. SKYRING discussed 'The use of indocyanin green as an index of hepatic function'. This was studied in a series of 93 subjects by a method requiring two blood samples exactly 10 minutes apart. A dose of $0.1 \mathrm{mg}$. $/ \mathrm{kg}$. was used. The results were expressed as percentage retention at 10 minutes.

In a group of 20 normal subjects and 24 patients with various medical diseases but without liver disease the values were all less than $20 \%$. In 48 patients with liver disease the values were all greater than $20 \%$ retention. There was a good correlation between severity of liver disease and percentage retention of the dye. There were no side effects. They concluded that indocyanin green is a reliable index of liver function and that values greater than $20 \%$ by this method represent evidence of liver disease.

R. W. BEAL and C. R. B. BLACKBURN discussed 'Studies on blood ammonia levels in patients with liver disease', with reference to the effect of nialamide. Nialamid (Niamid, Pfizer) has been shown to lower the level of blood ammonia in patients with cirrhosis of the liver, whether the elevation is endogenously or exogenously produced. Hepatic vein and renal vein catheterization, together with forearm and liver blood flow measurements were made to determine the mode of action. It was postulated from these results that Niamid may act by increasing hepatic blood flow, while maintaining a constant peripheral uptake, the net effect being to present more circulating ammonia to the liver for detoxication.

Observations on the 'Diagnosis and treatment of haemochromatosis' were presented by w. B. HENNESSY and BRUCE HALL. Thirty cases of haemochromatosis were studied. Twenty-one were men and nine were women. The age at which the disease was diagnosed varied from 19 to 80 years, with a peak incidence in the sixth decade. Two patients had a family history of haemochromatosis, three had suffered from severe anaemia, and five had a history of alcoholism. Hepatomegaly occurred in every patient; skin pigmentation was noted in 22 of these but diabetes mellitus occurred in only eight. The diagnosis was confirmed by either histological examination of the liver or serum iron studies or both. Gastric biopsy was performed in nine patients, and in all of these haemosiderin was demonstrated in the chief cells of the tubular glands.

Seventeen patients were treated by repeated venesection and in six the haemoglobin or serum iron levels eventually fell. To accomplish this, from $10 \mathrm{~g}$. to $26 \mathrm{~g}$. iron was removed.

Half the patients died within the follow-up period, but only four of these had been subjected to venesection. Five died from primary carcinoma of the liver.

All the surviving patients have experienced increased strength and well-being after phlebotomy. Skin pigmentation has lessened and the liver has become smaller. Liver function tests have improved. Serial liver biopsies showed diminution or complete disappearance of iron but the cirrhosis was unchanged.

B. F. VAUGHAN and W. R. PITNEY described the 'Radiological findings in 63 cases of megaloblastic anaemia'. They had reviewed the barium meal films in these cases without reference to the haematological findings.

Haematological diagnosis depended on the results of serum vitamin $\mathbf{B}_{12}$ concentrations, radioactive vitamin $B_{12}$ absorption tests, and gastric biopsy as well as the more usual diagnostic techniques and the response to therapy. Radiological gastric atrophy was demonstrated in 42 patients, 38 with pernicious anaemia and four with the malabsorption syndrome. All 42 patients suffered from vitamin $B_{12}$ deficiency.

In eight patients, megaloblastic anaemia occurred following gastric surgery, making a total of 50 abnormal barium meal examinations in the 63 patients. In one patient with folic acid deficiency megaloblastic anaemia due to malabsorption, in five with nutritional megaloblastic anaemia, and in two with megaloblastic anaemia associated with lactation, the barium meal findings were normal. In five patients with pernicious anaemia a normal barium meal was reported.

Sixteen of the 42 patients with radiological gastric atrophy demonstrated oesophageal regurgitation, six with associated hiatal hernia. The demonstration of a gastric abnormality in pernicious anaemia was fairly accurate $(90 \%)$; radiological examination was unable to assist in the identification of the mechanism of the anaemia in patients with a radiologically normal stomach or with partial gastrectomy.

PETER PARSONS reviewed a series of 16 patients with 'Gas cysts of the colon'. Their ages ranged from 30 to 70 years, and there were twice as many women. The left side of the colon was the most common site, particularly the splenic flexure and sigmoid.

All but three suffered from chronic bronchial asthma and at least 11 of these had been treated with corticosteroids for from one to five years. Ten cases presented with diarrhoea, the stools usually containing mucus, but appreciable bleeding occurred in three. One had subacute obstruction. When the cysts were low the diagnosis was established by sigmoidoscopy, but mistakes were made in four early cases before the observer was aware of the rather characteristic appearances. The higher cysts were detected by radiographs, the plain film showing the foam-like collections of gas shadows in the line of the colon in five of the cases in which they were done, and the barium enema gave confirmation in every case, but here again was misinterpreted in four of the early cases.

'Combined early endoscopy and radiology in the management of upper intestinal bleeding' was discussed by A. R. COOKE and A. P. SKYRING.

Forty-five consecutive and unselected patients admitted with upper intestinal bleeding were studied using a combination of early endoscopy and radiology. Endoscopy was performed in the ward within 24 hours of admission and was followed by a barium or gastrograffin meal in the Department of Radiology. The early diagnosis was 
confirmed at necropsy, operation, or a routine barium meal before discharge from hospital. An overall diagnosis rate of $82 \%$ was achieved within the first 24 hours. All gastric ulcers were detected by this method but duodenal ulcers were missed on two occasions.

Acute gastric erosions were seen in 10 patients. There remained a group of six patients in whom no diagnosis was made by either early or late investigation. The procedures were safe and in all cases an excellent view could be obtained with the gastroscope. It is hoped that early diagnosis places further management, especially if surgery is contemplated on a safer and surer clinical basis.

BKUCE BEVERIDGE described a 'Comparative study of various liver function tests in the differential diagnosis of jaundice'. A retrospective diagnosis was made on clinical, epidemiological, histological, and follow-up evidence in 53 patients with clinical jaundice, divided into five diagnostic groups: Extrahepatic obstruction, cholangiolitic hepatitis, parenchymal jaundice, drug cholestasis, and chronic liver disease. For each liver function test a scattergram showed the values obtained in the five diagnostic groups and from these the reliability of the test in differentiating the groups was judged.

Clinically jaundice due to chronic liver disease and drug-induced cholestasis rarely caused grave diagnostic problems, but the diagnosis of extrahepatic obstructive jaundice from cholangiolitic hepatitis and occasionally from parenchymal jaundice presented difficulty.

The serum alkaline phosphatase activity was not found to be a good indicator of extrahepatic obstruction. The most useful indicator of parenchymal jaundice was a thymol turbidity value greater than 3 units. Less valuable were markedly raised serum oxalo-acetic and pyruvic transaminase levels, while serum ornithine transcarbamylase and quinine oxidase levels were of little value. However, on biochemical grounds five patients appeared to have extrahepatic obstruction, though this was not in fact present.

The thymol turbidity results appeared independent of serum albumin and globulin concentration, which were helpful only in chronic liver disease.

\section{The September 1961 Issue}

\section{THE SEPTEMBER 1961 ISSUE CONTAINS THE FOLLOWING PAPERS}

Primary Crohn's disease of the colon and rectum J. S. CORNES and METTE STECHER

Difficulties in the diagnosis and management of unsuspected tuberculous enteritis and colitis E. J. MORAN CAMPBELL

Recurrent swelling of the parotid gland R. S. BRUCE PEARSON

$\mathrm{Co}^{58} \mathrm{~B}_{12}$ absorption (hepatic surface count) after gastrectomy, ileal resection, and in coeliac disorders D. J. FONE, W. T. COOKE, M. J. MEYNELL, and E. L. HARRIS

Comparative investigations on the activity of leucine aminopeptidase, glutamic oxalacetic transaminase, and alkaline phosphatase in serum B. G. MUNCK and $\mathrm{K}$. KJERULF

The effect of anticholinergic drugs on the electrolyte content of gastric juice D. W. PIPER and MIRJAM C. STIEL
Non-esterified fatty acids and lipoprotein lipase activity in patients with cirrhosis of the liver C. CHLOUVERAKIS and PETER HARRIS

The effect of vagotomy and pyloroplasty on the maximal acid response to histamine ALVIN M. GELB, IVAN D. BARONOFSKY, and HENRY D. JANOWITZ

The measurement of intestinal sounds in man and their relationship to serum 5-hydroxytryptamine B. G. ADAMS

The cardiac sphincter in the cat C. G. CLARK and J. R. VANE

Serum studies in man after administration of vitamin $A$ acetate and vitamin A alcohol OLIVER FITZGERALD, JAMES J. FENNELLY, and DANIEL J. HINGERTY

The problems of closed liver injuries R. H. B. MILLS

\section{Methods and techniques}

The microvilli of the small intestinal surface epithelium in coeliac disease and in idiopathic steatorrhoea MARGOT SHINER and M. S. C. BIRBECK

Copies are still available and may be obtained from the PUBLISHING MANAGER, BRITISH MEDICAL ASSOCIATION, TAVISTOCK SQUARE, W.C.1, price 17s. 6D. 